\title{
Robust control for nonlinear uncertain descriptor systems
}

\author{
Mourad Kchaou ${ }^{1}$ \\ ${ }^{I}$ Electrical Department, College of Engineering University of Hail KSA
}

\begin{abstract}
The present paper considers the sliding mode control (SMC)design problem for nonlinear uncertain descriptor systems. The goal is todesign an adaptivesliding mode controllerto drive the trajectories of the resulting closed-loop system onto a prescribed sliding surface and maintained there for all subsequent times. The appealing attributes of this approach include: (i) the closed-loop system exhibits a strong robustness against nonlinear dynamics and (ii) the control scheme enjoys the chattering-free characteristic. An example is provided to illustrate our control strategy.
\end{abstract}

Keywords: Input nonlinearity, Descriptor systems, LMI, sliding mode control

\section{Introduction}

Descriptor systems have been one of the major research fields of control community as a consequence of their flexibility for modelling real systems with constraints. They are convenient and natural representations to properly describe the behavior of many practical systems with both dynamic and static constraints. There have been a lot of work related to the stability, stabilization problems for descriptor systems; see $[1,2,3,9,12]$.

On the other hand, as one of the common methods, (SMC) has become a very effective approach in control engineering. It has proved to be an effective tool for coping with model uncertainties and non-linearities by taking advantage of the concepts of sliding mode surface design and equivalent control. During the past decades, various SMC approaches have been successfully applied for solving many practical control problems (see e.g. $[1,4,5,8,9])$. However, due to the physical limitation, the control input seems to have a non-linear character, such as sectors, saturation, dead-zone, and so on. Naturally originate from actuators in system realization, the non-linearities may deteriorate the system's stability and performances. So, their effects cannot be ignored in analysis of realization. Recently, attention has been paid to input nonlinearity [6,7], but few works has been undertaken on SMC for descriptor systems subjected to input nonlinearity.

In this paper, a novel robust (SMC) methodologyfor a class of uncertain nonlinear systems with mismatched uncertainties, and input nonlinearity, is addressed. The proposed results in this paper have the following advantages:

- Proposition of a new sliding function and establishment of sufficient conditions to ensure the robust admissibility of the corresponding sliding mode,

- Design of a sliding mode controller for the reaching motion such that all trajectories of the resulting closedloop system converge onto a prescribed sliding surface and maintain there for all subsequent times.

This paper is organized as follows. System description and preliminaries are provided in Section 2. Section 3 presents our design scheme.The simulation results of the proposed approach are detailed in in Section 4. Section 5 draws conclusions of thestudy.

Notations Throughout this paper, $\mathrm{X} \in \mathbb{R}^{\mathrm{n}}$ denotes the $\mathrm{n}$-dimensional Euclidean space, while $\mathrm{X} \in \mathbb{R}^{\mathrm{n} \times m}$ refers to the set of all $n \times m$ real matrices. The notation $X>0$ (respectively, $X \geq 0$ ) means that the matrix $X$ is real symmetric positive definite (respectively, positive semi-definite). The symbol (*) stands for matrix block induced by symmetry, $\operatorname{sym}(X)$ stands for $X+X^{T}$. Matrices are assumed compatible for algebraic operations if their dimensions are not explicitly stated. If not explicitly stated, all matrices are assumed to have compatible dimensions for algebraic operations.

\section{System description and preliminaries}

We consider a class of nonlinear descriptor system described by:

$$
E \dot{x}(t)=A(t) x(t)+B(\phi(u)+f(x(t)))
$$

where $x(t) \in R^{n}$ is the state, $u \in R^{m}$ is the control input and $\phi(u)$ is a nonlinear function of $u$. $f(t, x(t))$ represents the system nonlinearity and any model uncertainties in the system including external disturbances. Matrix $E \in \mathbb{R}^{\mathrm{n} \times \mathrm{n}}$ may be singular with $\operatorname{rank}(E)=q \leq n . A(t)=A+\Delta A(t)$ is time-varying system matrix. Matrices $A, B$ are constant with appropriate dimensions. Assume that uncertainties $\Delta A(t)$ is of the form $\Delta A(t)=M F(t) N$. where $M$ and $N$ are known real constant matrices and $F(t)$ is an unknown timevarying matrix function satisfying $F^{T}(t) F(t) \leq I$. In addition, $\phi(u)=\left[\phi_{1}\left(u_{1}\right), \phi_{2}\left(u_{2}\right), \cdots, \phi_{m}\left(u_{m}\right)\right]^{T}$, $\phi(0)=0$ is the nonlinear input vector. 


\section{Assumptions}

1. Matched nonlinearity $f(t, x(t))$ satisfies the inequality

$$
f(t, x(t)) \leq \eta(t, x(t))
$$

where $\eta(t, x(t))$ is a positive known vector-valued function

2. Nonlinear input $\phi(u)$ applied to the system satisfies $\phi(0)=0$ and

$$
u^{T} \phi(u) \geq \alpha u^{T} u
$$

where $\alpha$ is a positive constant.

Definition 1. [11] System $E \dot{x}=A x(t)$ (or the pair $(E, A)$ ) is said to be:

3. regular if $\operatorname{det}(s E-A) \neq 0$.

4. impulse free if $\operatorname{deg} \operatorname{det}(s E-A)=\operatorname{rank}(E)$.

5. admissible if it is regular, impulse-free and stable.

Lemma 1.[10] Let $M$ and $N$ be real matrices of appropriate dimensions. Then, for any $\Delta$ matrix satisfying $\Delta^{T} \Delta \leq I$ and scalar $\varepsilon>0$,

$$
\operatorname{sym}(M \Delta N) \leq \varepsilon M M^{T}+\varepsilon^{-1} N^{T} N
$$

\section{Main results}

In this section, an appropriate integral switching surface is designed such that the sliding mode dynamics restricted to the surface is admissible. Furthermore, a sliding mode control law is synthesized to guarantee that the system state trajectories are globally driven onto the predefined sliding surface and maintained them there for all subsequent time

\section{Integral sliding mode surface}

Define the following switching function

$$
s(t)=\mathbb{G} E x(t)-\mathbb{G}\left(E x_{0}+\int_{0}^{t}(A+B K) x(\theta) d \theta\right)
$$

and $K \in \mathbb{R}^{\mathrm{m} \times \mathrm{n}}$ is a real matrix to be designed and $\mathbb{G} \in \mathbb{R}^{\mathrm{m} \times \mathrm{n}}$ is a constant matrix satisfying $\mathbb{G} B$ is nonsingular. According to the sliding mode control theory, when the system trajectories reach onto the switching surface, we have $\dot{s}(t)=0$. Then the equivalent control law can be obtained as follows:

$$
\phi_{e}(u)=-K x(t)-f(x(t))
$$

Substituting (6) into (1), we obtain the following sliding mode dynamics:

where

$$
E \dot{x}=\bar{A}(t) x(t)
$$

$$
\bar{A}(t)=A(t)+B K, \quad \overline{\mathbb{G}}=I-B(\mathbb{G} B)^{-1} \mathbb{G}, \quad \bar{M}=\overline{\mathbb{G}} M,
$$

\section{2. sliding mode dynamics analysis}

In this subsection, we will develop a sufficient condition that ensures for sliding mode dynamics 7 to be robustly admissible.

Theorem 1. The sliding mode dynamics of (7) is admissible, if there exist matrices $S, P>0$, and $G_{j},(j=1,2)$ and a scalar $\varepsilon>0$ such that the following inequality holds :

$$
\left(\begin{array}{ccc}
\operatorname{sym}\left(G_{1} \bar{A}\right)+\varepsilon N^{T} N & P+S R^{T}-G_{1}+\bar{A}^{T} G_{2}^{T} & G_{1} \bar{M} \\
-\operatorname{sym}\left(G_{2}\right) & G_{2} \bar{M} \\
* & -\varepsilon I
\end{array}\right)<0
$$

Proof. The proof of this theorem is divided into two parts. The first one is concerned with the regularity and the impulse-free characterizations, and the second one treats the stability property of system (7). First we consider the nominal case of (7) (that is $\Delta \bar{A}=0$ ).

Since $\operatorname{rank}(E)=q \leq n$, there always exist two nonsingular matrices $\mathbb{M}$ and $\mathbb{N} \in \mathbb{R}^{n \times n}$ such that

$$
\mathbb{E}=\mathbb{M} E \mathbb{N}=\left(\begin{array}{ll}
I_{q} & 0 \\
0 & 0
\end{array}\right)
$$

Then $R$ can be characterised as $R=\mathbb{M}^{T}\left(\begin{array}{l}0 \\ \widehat{\Phi}\end{array}\right)$, where $\widehat{\Phi} \in \mathbb{R}^{(n-q) \times(n-q)}$ is any non-singular matrix.

We also define

$$
\hat{A}=\mathbb{M} \bar{A} \mathbb{N}=\left(\begin{array}{ll}
\hat{A}_{11} & \hat{A}_{12} \\
\hat{A}_{21} & \hat{A}_{22}
\end{array}\right), \hat{S}=\mathbb{N}^{T} S, P=\left(\begin{array}{ll}
\hat{P}_{11} & \hat{P}_{12} \\
\hat{P}_{21} & \hat{P}_{22}
\end{array}\right)
$$

Pre- and post-multiplying (9) by $\left[I \bar{A}^{T} 0\right]$ and its transpose, respectively, we can obtain easily that

$$
\operatorname{sym}\left(S R^{T} \bar{A}+P \bar{A}\right)<0
$$

Pre- and post-multiplying (12) by $\mathbb{N}^{T}$ and $\mathbb{N}$, respectively, and then using expressions 10 and 11 yields 


$$
\operatorname{sym}\left(\hat{S}_{21} \widehat{\Phi}^{T} \hat{A}_{22}\right)<0
$$

and $\bar{A}_{22}$ is thus non-singular. So, descriptor system (7) is regular and impulse free.

In the following, we will prove that system (7) is asymptotically stable. For this purpose, we construct a candidate Lyapunov function as:

$$
\boldsymbol{V}(x(t))=x^{T}(t) \operatorname{PEx}(t)
$$

Evaluating the derivative of $\boldsymbol{V}(x(t))$ along the solutions of system (7), it yields

$$
\dot{\boldsymbol{V}}(x(t))=x^{T}(t) P E \dot{x}(t)
$$

Defining $\xi(t)=\left[x^{T}(t) \dot{x}^{T}(t) E^{T}\right]^{T}$

From (7), the following equation holds for any matrices $G_{j}, j=1,2$ with the appropriate dimensions

$$
2 \xi^{T}(t)\left[G_{1}{ }^{T} G_{2}^{T}\right]^{T}[-E \dot{x}(t)+\bar{A} x(t)]=0
$$

Furthermore, noting $E^{T} R=0$, we can deduce that

Combining 18-20 yields

$$
2 \dot{x}^{T}(t) E^{T} R S^{T} x(t)=0
$$

$$
\dot{\boldsymbol{V}}(x(t)) \leq \xi^{T}(t) \Phi \xi(t)
$$

where

$$
\Phi=\left(\begin{array}{cc}
\operatorname{sym}\left(G_{1} \bar{A}\right) & P+S R^{T}-G_{1}+\bar{A}^{T} G_{2}^{T} \\
-\operatorname{sym}\left(G_{2}\right)
\end{array}\right)<0
$$

Hence, $\dot{\boldsymbol{V}}(x(t)) \leq-\lambda\|\xi(t)\|^{2}$ which implies that nominal singular system (7) is asymptotically stable. Consider now the uncertain case. By following the same procedure as used above it is easy to verify that

$$
\Phi+\operatorname{sym}\left(\left[G 1^{T} G_{2}^{T}\right]^{T} \bar{M} F(t) N\right)<0
$$

Then, according to Lemma 1, inequality (9) holds using the Schur complement. This completes the proof.

\section{3. sliding mode dynamics synthesis}

Here, our goal is to design gains $K$ in (5) such that sliding mode dynamics (7) is robustly admissible.

Based on Theorem 1, we suggest the following result:

Theorem 2. Given $\lambda$ a positive scalar. The sliding mode dynamics of (7) is admissible, if there exist matrices $S$, $P>0, Y$ and $X$ and positive scalars $\varepsilon$ and $\epsilon$ such that the following inequalities hold :

$$
\left(\begin{array}{cccccc}
\operatorname{sym}(A X+B Y) & \bar{P}-X+\lambda(A+B Y)^{T} & \bar{M} & X N & \bar{S} R^{T} & 0 \\
& -\lambda \operatorname{sym}(X) & \lambda \bar{M} & \lambda X N & 0 & X \\
& * & -\varepsilon I & 0 & 0 & 0 \\
& * & * & -\varepsilon I & 0 & 0 \\
* & * & * & -\epsilon I & 0 \\
& * & * & * & * & -\epsilon I
\end{array}\right)<0
$$

Proof: Under the conditions of Theorem 1, a feasible solution satisfies condition- $\operatorname{sym}\left(G_{2}\right)<0$, which implies that, $G_{2}$ is nonsingular. Let $G_{2}=\lambda G_{1}$ and $X=G_{1}^{-1}$. Checking a congruence transformation to (9) by $\operatorname{diag}(X, X, I)$ and using the fact that

$$
2 \operatorname{sym}\left(\left[\begin{array}{c}
X^{T} S \\
0 \\
0
\end{array}\right]\left[\begin{array}{lll}
0 & R^{T} X & 0
\end{array}\right]\right)<\epsilon\left[\begin{array}{c}
X^{T} S \\
0 \\
0
\end{array}\right]\left[\begin{array}{c}
X^{T} S \\
0 \\
0
\end{array}\right]+\epsilon^{-1}\left[\begin{array}{c}
0 \\
X^{T} R \\
0
\end{array}\right]^{T}\left[\begin{array}{c}
0 \\
X^{T} R \\
0
\end{array}\right]^{T}
$$

inequality (24) holds using Schur complement.

\section{SMC law synthesis}

After establishing the appropriate switching surface (5), we will focus on designing a sliding mode controller to drive the system trajectories onto the sliding mode $s(t)=0$ even though uncertain and input nonlinearity are presented. To achieve the control objective, the input $u(t)$ is designed as follows:

where $\Psi$

$$
u(t)=-\hat{\alpha}(t)(\Psi+\chi) \frac{s(t)}{\|s(t)\|}
$$

and $\chi>0$, is a small constant.

$$
\Psi=\left\|(\mathbb{G} B)^{-1} \mathbb{G}\right\|\|M\|\|N\|\|x(t)\|+\|K x(t)\|+\eta(t, x(t))
$$

Next, in the following theorem, it is shown that the sliding motion will be driven onto the specified switching surface $s(t)=0$ and be maintained there subsequently.

Theorem 1. If the control input $u(t)$ is designed as (23)-(24), then the trajectory of the system (1) converges to the sliding surface $s(t)=0$. 
Proof.

Consider the following Lyapunov function:

where $\tilde{\alpha}(t)=\hat{\alpha}^{-1}(t)-\alpha$

$$
V_{s}(t)=\frac{1}{2} s^{T}(t)(\mathbb{G} B)^{-1} s(t)+\frac{1}{2 \kappa} \tilde{\alpha}^{2}(t)
$$

Without loss of generality, we can choose $\mathbb{G}=B^{T} X_{0}$, where $X_{0}$ is a positive definite matrix. So $\mathbb{G} B=B^{T} X_{0} B$ is nonsingular.

According to ?, we get

$$
\dot{s}(t)=\mathbb{G}((\Delta A(t)-B K) x(t)+B(\phi(u(t))+f(x(t))))
$$

Thus, taking the derivative of $V_{s}(t)$ and considering the above equation, we have

$$
\begin{gathered}
\dot{V}_{s}(t)=s^{T}(t)(\mathbb{G} B)^{-1} \dot{s}(t)-\frac{1}{\kappa} \tilde{\alpha}(t) \frac{\dot{\hat{\alpha}}(t)}{\hat{\alpha}^{2}(t)} \\
=s^{T}(t)\left((\mathbb{G} B)^{-1} \mathbb{G}(\Delta A(t)-B K) x(t)+\phi(u(t))+f(x(t))\right) \\
\leq(1-\hat{\alpha}(t) \tilde{\alpha}(t) \Psi\|s(t)\| \\
\leq(t)) \Psi\|s(t)\|+s^{T}(t) \phi(u(t))
\end{gathered}
$$

Using (23) and assumption 3 , it can be derived that

$$
\begin{gathered}
u^{T}(t) \phi(u)=-\hat{\alpha}(t)(\Psi+\chi) \frac{s^{T}(t)}{\|s(t)\|} \phi(u(t)) \geq \alpha u^{T}(t) u(t) \\
=\alpha \hat{\alpha}^{2}(t)(\Psi+\chi)^{2}
\end{gathered}
$$

Since $(\Psi+\chi)>0$, we get

Substituting (29) into (27), we obtain

$$
s^{T}(t) \phi(u(t)) \leq-\alpha \hat{\alpha}(t)(\Psi+\chi)\|s(t)\|
$$

Noting that $\alpha \hat{\alpha}(t)+\hat{\alpha}(t) \tilde{\alpha}(t)=1$ and $\hat{\alpha}(t)>0$, it is easy to verify that

$$
\dot{V}_{s}(t)<0, \quad \forall t>0
$$

This means the system trajectories converge to the predefined sliding surface and are restricted to the surface for all subsequent time. This completing the proof.

\section{Simulation Results}

Now, we demonstrate the applicability of the main results by means of a simulation example.

\section{Example 1:}

Consider the switched systems (1) with the following parameters:

$$
E=\left[\begin{array}{lll}
1 & 0 & 0 \\
0 & 1 & 0 \\
0 & 0 & 0
\end{array}\right], A=\left[\begin{array}{ccc}
-2.7 & 0 & 0.3 \\
-0.2 & -3 & 0 \\
0.11 & 1.74 & -1
\end{array}\right], B=\left[\begin{array}{l}
1 \\
0 \\
0
\end{array}\right],
$$

Assume that the uncertain matrices are as follows:

$$
M=\left[\begin{array}{lll}
0.5 & -0.5 & 0.5
\end{array}\right], \quad N=\left[\begin{array}{lll}
1 & 1 & 1
\end{array}\right] .
$$

Our aim is to design an SMC in the form of 2 such that the sliding mode dynamics is robustly admissible. In the simulation, the design parameters are chosen as

$$
\mathbb{G}=\left[\begin{array}{lll}
1 & 1 & 1
\end{array}\right]
$$

By solving LMI (?) we can obtain a feasible solution with the following parameters:

The associate controller gains are

$$
P=\left[\begin{array}{ccc}
0.9714 & -0.0244 & -0.0315 \\
-0.0244 & 0.8634 & -0.0708 \\
-0.0315 & -0.0708 & 0.9090
\end{array}\right],
$$

$$
K=\left[\begin{array}{lll}
-0.7300 & 0.4471 & 0.6077
\end{array}\right]
$$

The existence of a feasible solution shows that there exists a desire sliding surface in (5) such that the resulting sliding mode dynamics in (7) is admissible.

The remaining task is to design a sliding mode controller such that the system trajectories can be driven onto the predefined sliding surface and maintained there for all subsequent time. For simulation purposes, we uncertain matrix function $F(t)=0.8+0.2 \sin (t)$ and

$$
f(x)=1.7 x_{1}^{2} \cos \left(x_{2}\right)+0.5 \sqrt{\left|x_{1} x_{3}\right|}, \quad \phi(u)=(0.5+0.3 \cos (u)) u,
$$

With $\chi=0.5$ and $\kappa=0.01$, the adaptive SMC law can be designed according to (2)-(?).

To prevent the control signal from chattering, we replace $\frac{s(t)}{\|s(t)\|}$ with $\frac{s(t)}{0.05+\|s(t)\|}$.

The simulation results depicted in figure (1) show that: 


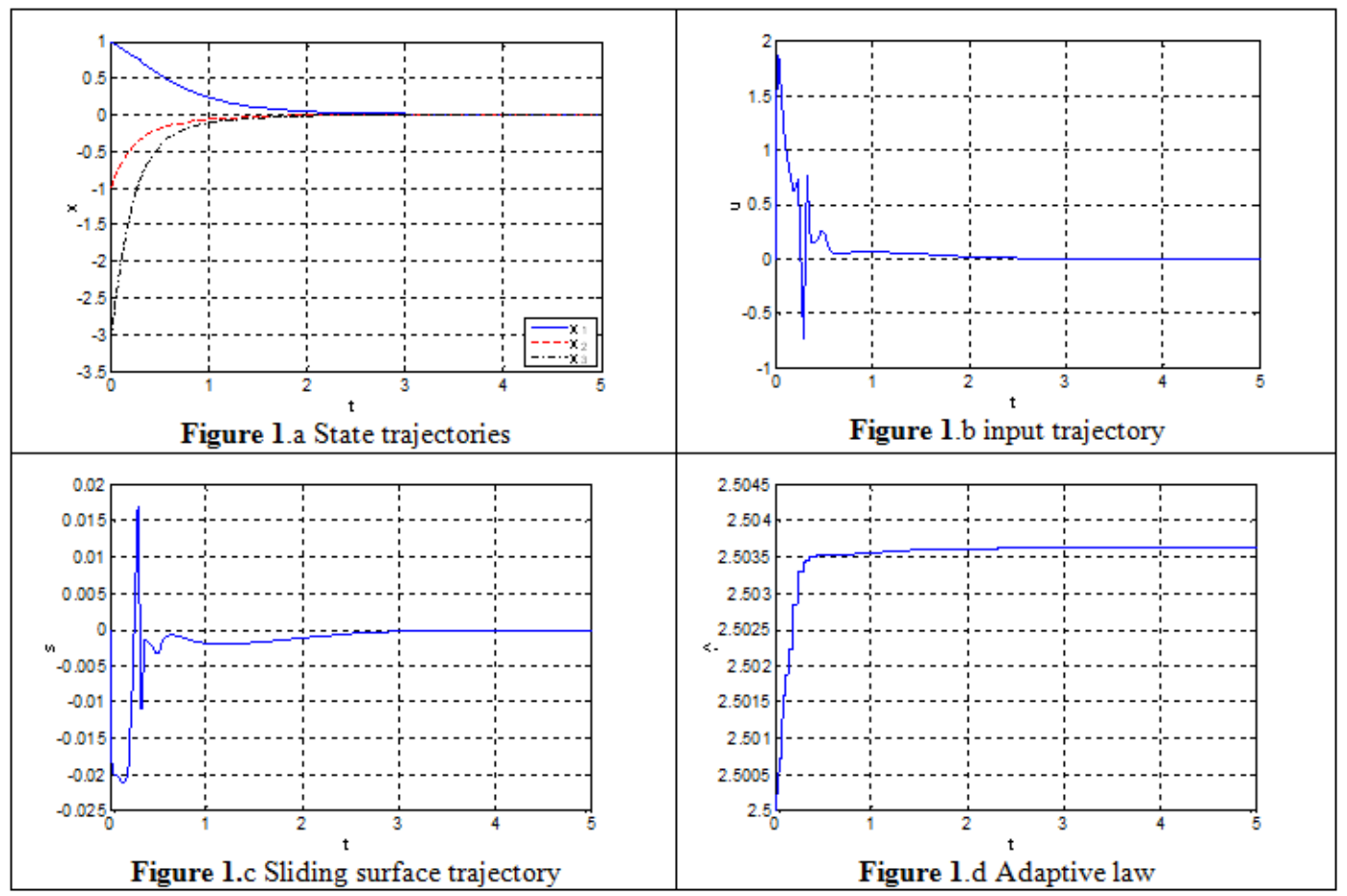

Figure 1: Simulation results

for initial condition $x_{0}(t)=[-1,1,1.5]^{T}$, Figures (1a)-(1d) depict respectively, the system state trajectories, the control input, the resulting sliding surface and the adaptive law when the SMC is applied. We observe that the system is stable despite the presence of actuator nonlinearity, parameter uncertainties and external disturbances. The proposed scheme can obtain better convergence performance by driving the system trajectories to the specified sliding surface asymptotically instead of to some neighbor of the surface.

\section{Conclusion}

A novel adaptive sliding model controller is proposed in this paper for stabilizing uncertain nonlinear descriptor systems nonlinear input containing sector nonlinearities. An integral sliding function is proposed and a sufficient condition is derived to guarantee that the sliding mode dynamics is robustly admissible. Moreover, an adaptive (SMC) law is designed such that the trajectories of the resulting closed-loop system can be driven onto a prescribed sliding surface and maintained there for all subsequent time. Finally, a simulation example is presented to demonstrate the effectiveness of the proposed method. The proposed controller shows that has the ability to eliminate the model uncertainties and to reduce the chattering on the sliding surface

\section{First ref removed}

\section{References}

[1]. Cui, W.; Fang, J.; Shen, Y. \& Zhang, W."Dissipativity analysis of singular systems with Markovian jump parameters and modedependent mixed time-delays",Neurocomputing, 2013, 110, 121-127

[2]. Feng, Z.; Lam, J. \&Gao, H.” $\alpha$-Dissipativity analysis of singular time-delay systems”Automatica, 2011, 47, 2548-2552

[3]. Chang, J. "Dynamic output feedback integral sliding mode control design for uncertain systems", International Journal of Robust and Nonlinear Control, 2012, 22, 841-857

[4]. Li, F., Wu, L., Shi, P. and Lim, C. "State estimation and sliding mode control for semi-Markovian jump systems with mismatched uncertainties," Auomatica (51), 2015, pp. 385-393.

[5]. Liu, L., Pu, J., Song, X., Fu, Z. and Wang, X. "Adaptive sliding mode control of uncertain chaotic systemswith input nonlinearity," Nonlinear Dyn (76:1857-1865), 2014.

[6]. Nguyen, T., Liao, T. and Yan, J. "Improved adaptive sliding mode control for a class of uncertain nonlinear systems subjected to input nonlinearity via fuzzy neural networks," Mathematical Problems in Engineering (2015), 2015, pp. 1-13.

[7]. Wu, L. \& Ho, D. "Sliding mode control of singular stochastic hybrid systems", Automatica, 2010, 46, 779-783

[8]. Ding, Y.; Zhu, H. \&Zhong, S. "Exponential stabilization using sliding mode control for singular systems with time-varying delays and nonlinear perturbations", Communications in Nonlinear Science and Numerical Simulation, 2011, 16, 4099-4107

[9]. I.R Petersen. "A stabilization algorithm for a class of uncertain linear systems". Systems \&Control Letters, 8:35-357, 1987.

[10]. L Dai. "Singular Control Systems", volume 118 of Lecture Notes in Control and Information,Sciences. Springer, New York, 1989.

[11]. G.R Duan. "Analysis and Design of Descriptor Linear Systems". Springer Verlag, New York,2010. 